\title{
PSF Corrected Reconstruction in Soft X-ray Tomography (SXT)
}

\author{
Axel Ekman ${ }^{1}$, Tia E. Plautz ${ }^{1}$, Jian-Hua Chen ${ }^{1}$, Gerry McDermott ${ }^{1}$, Mark A. Le Gros ${ }^{1}$ and Carolyn \\ Larabell $^{1}$ \\ 1. Department of Anatomy, University of California, San Francisco, CA, USA
}

Soft x-ray tomography (SXT) refers to the $\mathrm{x}$-ray microscopy technique in which tomographic imaging is done using low-energy x-rays. In particular, the energy range of the photons lie within the "water window", i.e., between the K-absorption edges of oxygen $(2.34 \mathrm{~nm} ; 530 \mathrm{eV})$ and carbon (4.4 $\mathrm{nm} ; 280 \mathrm{eV}$ ) [1]. This region of $\mathrm{x}$-ray energies is especially suitable for imaging biological samples as, as the name suggests, water is relatively transparent to the x-rays and the contrast of the image comes from the natural absorption of the bio-molecules.

Biological soft $\mathrm{x}$-ray microscopes are analogous to conventional light microscopes, this means that SXT is diffraction limited, with a resolution $r \propto \lambda / N A$ and a depth of field DOF $\propto \lambda / N A^{2}$, where $\lambda$ is the wavelength of the illuminating light $N A$ the numerical aperture of the objective lens. Compared to through focus imaging, where a high resolution if achieved with a high $N A$ optical system, the best result in transmission tomography is achieved when the DOF spans the whole sample. If the energy would not be restricted, a sufficient DOF and a good resolution could be obtained by increasing the energy and decreasing the $N A$, but as the energy is restricted by the water window, an improvement in resolution is coupled with a decrease in maximum sample size.

Traditionally, the image formation in SXT has been based on ideal projection, in which the in-tensity of light rays is attenuated according to the Beer-Lambert law. Although this is a good approximation for highly elongated point spread functions (PSF), the actual image formation may differ substantially from this "ideal" model. When imaging samples that are larger than the DOF the PSF depends on the relative position along the optical axis. In the field of electron tomog-raphy, there is a known approximative solution to this depth-dependent inversion problem called the defocus-gradient correction [2], where both the forward and backward projections in the tomo-graphic reconstruction are corrected for depthdependent defocus.

Recently a model for SXT tomography bt Oton et al. [3] gives the basis to apply the same kind of correction in SXT. The feasibility and practical example of this was shown in Ref. [4], were a depth independent correction lead to higher contrast in the obtained reconstruction. The model is based on a mixture of coherent and incoherent assumptions for the illumination, in which the measured projections result from the attenuated light passing through the sample, smeared by the PSF of the objective lens. The incoherent assumption comes from assuming linear transfer such that there exists an impulse response function $h_{z}$, such that the field intensity at the image plane $I_{\text {im }}\left(\boldsymbol{x}_{\mathrm{im}}\right)$, can be expressed by linear transport of an unattenuated field. On the other hand, for small $N A$, the local field propagation can be done by assuming a parallel wave (coherent approximation). By constructing the derivative of $I_{\text {im }}^{z}$ with respect to $z$ from finite difference they obtain a model for the image formation in the form of

$$
I_{\mathrm{im}}\left(\boldsymbol{x}_{\mathrm{im}}\right)-I_{\mathrm{im}}^{0}\left(\boldsymbol{x}_{\mathrm{im}}\right)=\int_{\mathbf{R}}\left(-\mu\left(\boldsymbol{x}_{z}, z\right) I\left(\boldsymbol{x}_{z}, z_{0}\right) \mathrm{e}^{\int^{z}-\mu\left(\boldsymbol{x}_{t}, t\right) \mathrm{d} t}\right) * *\left|h_{z}\left(\boldsymbol{x}_{z}\right)\right|^{2} \mathrm{~d} z,
$$

where $\mu$, is the LAC of the specimen, $\boldsymbol{x}_{z}$ represents sets of coordinates, corresponding to the planes perpendicular to the optical axis at position $z$. This gives us the mathematical description of the 
image formed at the detector $I_{\mathrm{im}}$, where $I^{0}{ }_{\mathrm{im}}$ is the recorded image with no sample present, also known as the flat field.

There is no known direct inversion for Eq. (1) but by constructing the image as described in Ref. [3] but taking the finite difference approximation on the normalized absorption images, $y=-\log \left(I_{\mathrm{im}} / I_{\mathrm{im}}^{0}\right)$, we can produce a linear approximation to the image formation. With the linear ap-proximation of the image formation we formulate the measurement in terms of a linear transform on the (unknown) discrete representation of the LAC distribution $\boldsymbol{x}$ such that $\boldsymbol{y}=\boldsymbol{A x}$, where the matrix elements $\boldsymbol{A}_{i j}$ represent the contribution of $j$ th voxel in the LAC distribution on the projec-tion on the $i$ th detected pixel, now incorporating the effect of the PSF. In this way we have a linear model for the forward projection of the model and thus the reconstruction can be obtained by any of the various iterative reconstruction schemes available.

To illustrate the proof of concept, we consider an exaggerated test case with a broad PSF (Rayleigh resolution of 5 pix), while keeping the depth of field relatively short (87 pix) as compared to the size of the reconstruction $\left(256^{2}\right)$. In Fig. 1 we show the resulting reconstructions of two example measurements: one in-focus, where the PSF was centered on the center of rotation, and one out-of-focus, where the focal spot was shifted towards the edge of the image, aswell as an example from an actual dataset.

The incorporation of the PSF in the image formation is an essential step forward in improving the capability of modern SXT microscopes. It enables for measurement setup, where the resolution of the reconstruction is not limited by the conventional trade-off between sample size and resolution, as e.g. multiple through-focus images can easily be incorporated in the reconstruction. The linear nature of the approximation, makes the problem solvable with a large amount of different methods, and e.g. prior information is easily incorporated in the reconstruction scheme.

\section{References:}

[1] D Attwood. Soft x-rays and extreme ultraviolet radiation: principles and applications. Cambridge University Press, 2007.

[2] Grant J Jensen and Roger D Kornberg, Ultramicroscopy 84 (2000), p. 57.

[3] J Otón et al, Journal of Structural Biology 178 (2012), p. 29.

[4] J Otón et al, Biomedical Optics Express 7 (2016), p. 5092.
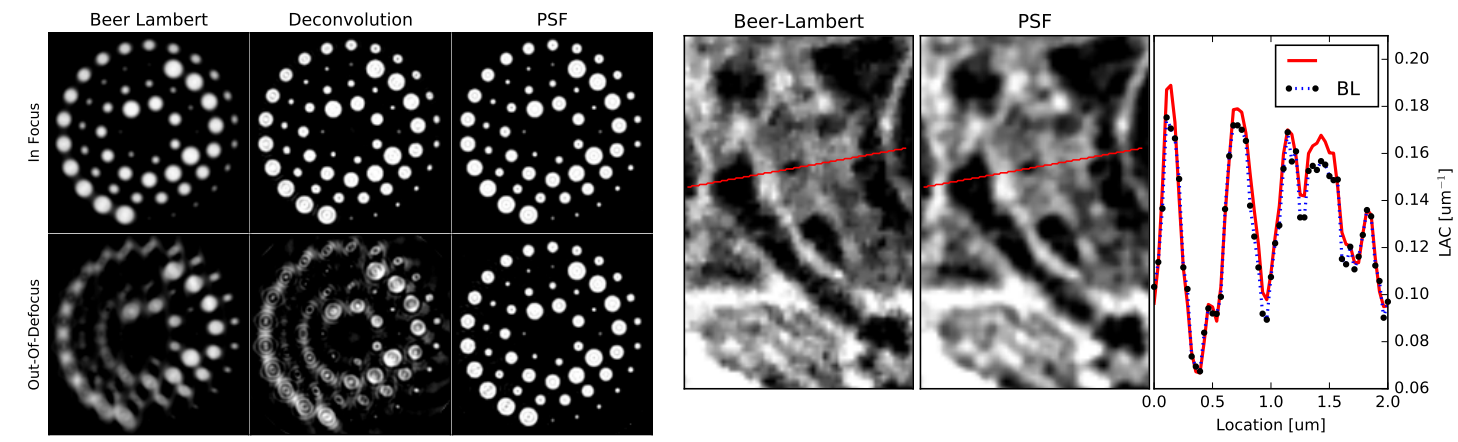

Figure 1. (left) Phantom reconstructions using BL approximation, wiener deconvolution and the PSF model for the two different PSFs. (right) Detail of an example reconstruction of a biological sample using two different forms for the projection matrix AAA. 